\title{
A Proposal for Routing Protocol for FANET: A Fuzzy System Approach with QoE/QoS Guarantee
}

\author{
Jorge Souza $\left(\mathbb{D},{ }^{1}\right.$ José Jailton $\left(\mathbb{D},{ }^{2}\right.$ Tássio Carvalho, ${ }^{2}$ Jasmine Araújo $\mathbb{D}^{\circ},{ }^{2}$ and Renato Francês ${ }^{2}$ \\ ${ }^{1}$ Ciberespacial Institute, Federal Rural University of the Amazon (UFRA), Belém, PA, Brazil \\ ${ }^{2}$ Institute of Technology, Federal University of Pará (UFPA), Belém, PA, Brazil \\ Correspondence should be addressed to Jorge Souza; jams.souza@gmail.com
}

Received 21 May 2019; Revised 30 September 2019; Accepted 4 November 2019; Published 23 November 2019

Guest Editor: Zeeshan Kaleem

Copyright (c) 2019 Jorge Souza et al. This is an open access article distributed under the Creative Commons Attribution License, which permits unrestricted use, distribution, and reproduction in any medium, provided the original work is properly cited.

The flying ad hoc network (FANET) has emerged as an alternative access technology for regions that have no fixed infrastructure or are hard to reach. This new type of network is composed of devices called unmanned aerial vehicles (UAVs) that communicate with each other, but there is no specific routing protocol to FANET applications that allows efficient communication between these devices. This paper proposes a FANET adaptive routing protocol based on the fuzzy system. The validation of the FANET adaptive protocol was performed through simulation using Network Simulator version 2 (NS-2) and, it was assessed by quality of service (QoS) and quality of experience (QoE) metrics.

\section{Introduction}

In recent years, there has been a great deal of motivation in the search for new wireless communication mechanisms, with great growth due to new technologies or configurations in aerial technologies, to monitoring hard-to-reach areas or in case of disaster situations. In this context, FANETs (flying ad hoc networks) emerged, which are a type of ad hoc network configuration consisting of unmanned aerial vehicles (UAVs). UAVs are responsible for monitoring a specific area by capturing images and sending them to a base ground station [1] in a process known as UAV-to-ground (U2G) communication.

In this context of FANETs, new challenges arise unlike the traditional infrastructure and cable challenges: (1) positioning the UAVs in a most appropriated way to monitor regions, minimizing costs and maximizing the performance of network; (2) reduce the negative effects of UAVs high mobility; and (3) traditional routing protocols are not capable of handling, in an efficient fashion, the flying networks, especially flying ad hoc networks, due to features such as high node mobility and topology changes. This can compromise communication between UAVs and network performance. Due to this, it is important to define a strategy to ensure adequate communication under these conditions, providing resources that guarantee satisfactory and intelligent performance to reduce the selected challenges [2].

Another important point to consider in FANET/UAV scenarios is that most mobile devices have an average flight time of approximately 30 minutes $[3,4]$ due to limited battery capacity. This fact draws the following conclusion: a node, with a low battery charge, will stop being part of the aerial network due to its "death," requiring the network to autoconfigure and restructure and reorganize its topology, avoiding damages that could affect aerial wireless communication. Because of this, it is necessary to determine and/ or predict which devices have low flight autonomy or are close to being incapacitated, in order to minimize the impacts on communication quality.

UAVs need to have sensors attached to them to capture real-time images of a certain area, and because of this, it is necessary to evaluate the quality of the data and video collected, which can be done by using QoE (quality of experience), that have equivalent direct measurements related to the evaluation of multimedia streams, from the user perception point of view, corroborating and complementing traditional QoS (quality of service) metrics, which evaluate objective measurements from the network point of view as 
throughput, delay, etc., but do not reflect the final user experience with the video streams nor the final quality of the received video [5].

As already commented, flight range and mobility are influential factors that can severely alter the topology of the aerial network. Because of this, it is of fundamental importance to build a new routing protocol that can consider these factors and exert a mutual interaction between them and the topological changes of the network, especially since currently there are no specific routing protocols for these ad hoc network scenarios beyond traditional routing protocols that date back to the emergence of the Internet such as Adhoc On Demand Distance Vector (AODV) and optimized link state routing (OLSR) that are neither adequate nor efficient in these scenarios [6].

Taking the numerous problems encountered in FANETS and air network scenarios, this paper proposes a routing protocol adapted and implemented for these scenarios created through a fuzzy system to enable the best communication path between UAVs (a process known as UAVto-UAV communication-U2U). The new routing protocols will find the best route, with the best connection and longest durability, improving network performance.

The paper is distributed as follows: Section 1, presented here, contextualized the work and introduced its contribution. Section 2 discusses the related works and compares them. Section 3 presents the state of the art of FANET applications. Section 4 describes the proposed new routing protocol and the details of the implemented system. Section 5 discusses the performance evaluation of the proposed routing protocol, which is sequentially complemented by Section 6 which concludes the work and presents its main contributions.

\section{Related Work}

This section examines the related work on the routing protocol flying ad hoc networks (FANETs). The aim is to demonstrate some gaps in related work and how the proposed routing protocol addresses them.

In paper [7], through simulations in Network Simulator 3 (NS-3), performance of AODV, OLSR, and HWMP protocols in FANETs was compared. The protocols were evaluated using the QoS metrics and Gauss-Markov mobility model. Despite the comparison, the paper proposed no improvement to the protocols.

In paper [8], the authors devise a new mechanism for data routing based on localization in GPS-denied or GPSchallenged areas. This mechanism relies on a weighted centroid localization technique, where the position of unknown UAV nodes is calculated by means of fuzzy logic. This paper does not take into account that changes in topology require new packet routing or the flight autonomy of each drone.

In paper [9], the authors investigated a routing protocol called predictive OLSR (P-OLSR), which is an extension of the traditional OLSR protocol. The authors compared the P-OLSR with OLSR by means of QoS metrics. However, although the paper put forward a new routing protocol, it did not compare it with the other routing protocols and failed to evaluate it with the QoE metrics.

In paper [10], the authors recommended an energy-efficiency algorithm for drones that included the parameters of the communication channel. This system reduced energy consumption, but even though there were a number of changes in the topology, the network was unable to identify new routes in an effective way and thus maintain the required level of quality.

In paper [11], the authors carried out a survey of the routing protocols for VANET and FANET. Their paper discusses the use of optimization techniques (particle swarm optimization, ant colony optimization, and bee colony optimization) to improve the routing performance but did not employ flight autonomy as a parameter or take QoE metrics into account.

In paper [12], the authors discuss the use of hop-by-hop communication between the drones as a means of increasing the network coverage area. However, the paper does not discuss to what extent the changes in the topology can have an effect on the quality of transmission, while also failing to address the need for an efficient routing protocol.

In paper [13], the authors introduced the use of the multiple path OLSR (MP-OLSR) routing protocol into FANET applications, especially in wildfires, to increase the chances of rescuing victims. The work considered scenarios with different speed ranges but did not address the flight autonomy problem.

In paper [14], the authors proposed the use of continuous Hopfield neural network ( $\mathrm{CHNN})$ to optimize the routes found by the dynamic source routing (DSR) protocol so that they would adapt to the high-speed movement of the FANET nodes. Simulation using NS-3 showed that the optimized DSR protocol improved the indicators such as end-to-end average delay, throughput, and packet delivery ratio. The authors did not address QoE and QoS metrics.

The authors in [7-14] examine the techniques that are used in FANET, but none of them set out a routing protocol that provides an effective response to the changes in the network topology. These papers do not address flight autonomy of the drones either and do not use a computer intelligence system for decision-making.

The proposed routing protocol, unlike those addressed in the related work presented in the literature, considers the needs and challenges of a FANET. Table 1 shows the related work that broadly addresses FANET routing and its solutions. This paper proposes an alternative approach to routing protocols already used in FANET by adopting fuzzy systems. The proposed routing protocol was validated by QoE metrics.

\section{Flying Ad Hoc Network (FANET)}

The increasing use of mobile ad-hoc networks (MANETs), vehicular ad hoc networks (VANETs), and wireless sensor networks, has led them to use new devices capable of moving and flying autonomously, generating more complex systems.

In FANET, the devices are generally referred to as unmanned aerial vehicles (UAVs). The use of UAVs has 
TABLE 1: Related work.

\begin{tabular}{|c|c|c|c|}
\hline Proposal & $\begin{array}{c}\text { Flight } \\
\text { autonomy }\end{array}$ & Decision strategy & Proposal focus \\
\hline [7] & No & Using the Gauss-Markov mobility model & $\begin{array}{c}\text { Evaluate the traditional routing protocol in } \\
\text { FANETs }\end{array}$ \\
\hline [8] & No & $\begin{array}{l}\text { Mechanism for data routing based on } \\
\text { localization }\end{array}$ & The position for UAV device \\
\hline [9] & No & Extension of the OLSR protocol called P-OLSR & Compare P-OLSR with OLSR \\
\hline [10] & No & Energy-efficiency algorithm & Reduce the energy consumption \\
\hline [11] & No & Survey of the routing protocols & $\begin{array}{l}\text { Discuss the use of optimization techniques to } \\
\text { improve the routing performance }\end{array}$ \\
\hline [12] & No & Discuss the hop-by-hop communication & $\begin{array}{c}\text { Number of UAVs necessary to network coverage } \\
\text { area }\end{array}$ \\
\hline [13] & No & Routing based on multiple paths & $\begin{array}{l}\text { Prove the efficiency of MP-OLSR in monitor } \\
\text { wildfire areas to increase the saving chances of } \\
\text { people victims of them }\end{array}$ \\
\hline [14] & No & CHNN-DSR protocol & $\begin{array}{l}\text { Optimize routes to adapt them to high-speed } \\
\text { movement of nodes }\end{array}$ \\
\hline $\begin{array}{l}\text { Current } \\
\text { proposal }\end{array}$ & Yes & Routing protocol with fuzzy logic system & $\begin{array}{c}\text { Select the best routing communication considering } \\
\text { flight autonomy with mobility and RSSI (received } \\
\text { signal strength indicator) }\end{array}$ \\
\hline
\end{tabular}

created new ways of operating innovative applications, by introducing a new type of network paradigm known as FANETs.

The networks differ from their traditional predecessors (MANETs in this case) as a result of the high mobility of UAVs, their greater connectivity, expansion in application areas, etc. In this respect, FANETs can generalize and extrapolate the topologies from $2 \mathrm{D}$ to $3 \mathrm{D}$ through a freemotion scheme, owing to the ability of the drones to fly independently in three-dimensional space. This new context has attracted researchers and industry as well as providing a driving force for real-life applications.

FANETs are generally used to provide connectivity to hard-to-reach places in regions where there have been natural disasters or even for military applications. After a catastrophic event (such as an earthquake, hurricane, tsunami, and dam breach), traditional network infrastructures can suffer damage and be subject to automatic shutdowns. However, through a FANET configuration, they could be employed to restore and provide sufficient connection and communication to the network in isolated areas. Besides, UAVs could be equipped with cameras and other types of sensors and devices to provide a constant aerial view and thus help rescue crews and firefighters to save lives.

In extensive coverage areas, it may be impracticable to establish direct communication from the UAVs to the base station on the ground at certain times. However, this problem can be overcome through hop-by-hop communication, which requires the use of a routing protocol to discover the best route/path from the source to the final destination [15].

3.1. Problem Statement and Major Contributions. The high mobility of UAVs means that network topology can change over time, so discovering and maintaining routes becomes one of the main issues to address [16]. For this reason, the focus of this paper is to establish a specific FANET protocol that can perform this task more efficiently.

Routing protocols are responsible for finding, establishing, and maintaining routes between two nodes that wish to communicate. These protocols should minimize overhead and bandwidth consumption.

A routing protocol that targets FANETs is more complex than fixed network protocols; this is due to several features of these networks such as their dynamic topology algorithm, mutual interference, restricted power, and the limited resources available in the UAVs.

In a FANET, given UAV mobility, it may happen that one aircraft is not close enough to another to establish communication, so it will need to use routing information to choose an alternate route. This communication can be done in multiple hops through the collaboration of intermediate nodes; that is, communication is not restricted to the radius of action of each device individually, but the sum of the radius of action of all devices (Figure 1).

The mobility of UAVs and their spatial arrangement are also very important for determining the communication routes. As a result of the movement, these routes are usually rearranged so that the interconnection between the UAVs can be continued. For this reason, the routing must be carried out dynamically by increasing the autonomy of the UAVs and reducing the delay in data delivery between a source node and a destination node [17].

Another main contribution of this paper is the adoption of a new communication network model used to provide connectivity in regions that are difficult to reach on land (especially regions after natural disasters). FANETs are easily established, as they are easily moved to a new region.

The frequent updating of the control information can ensure more accurate information; however, there is a need for a greater use of energy since this reduces the autonomy of the nodes. For this reason, this paper proposes a specific routing protocol for FANETs where the received signal 


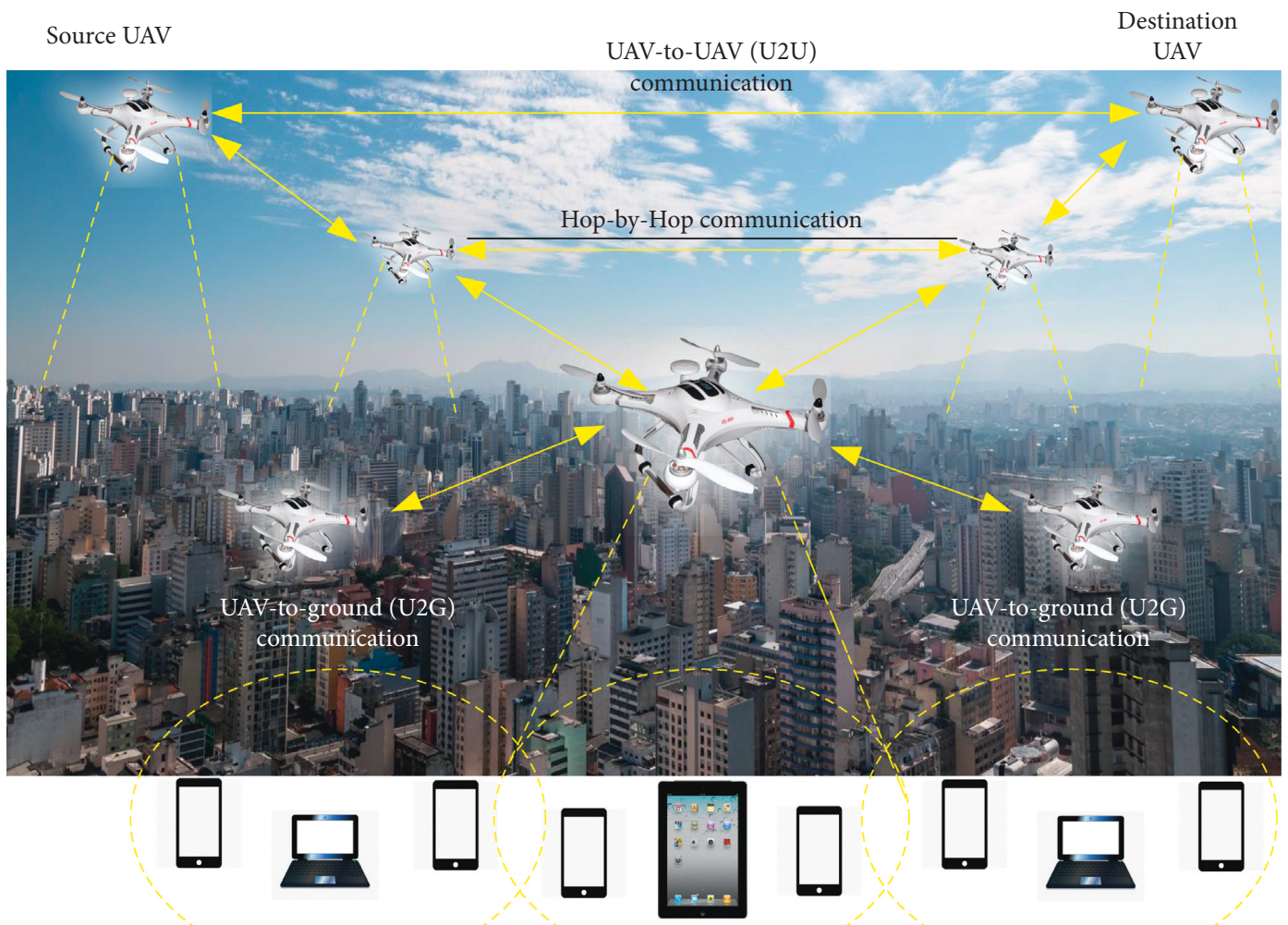

Figure 1: Hop-by-hop communication in FANET.

strength indicator (RSSI), mobility level, and in particular the flight autonomy of each UAV are employed as decisionmaking metrics to ensure quality of service and quality of experience for the network.

This paper also proposes, as a contribution, the use of a fuzzy system for the implementation of the routing protocol, with a set of inputs composed of information collected, in real time, from the network itself. The pieces of information collected are flight autonomy, mobility level, and RSSI. Based on such information, it is possible to establish communication routes that will remain active for a longer period. The objective is to discover routes with high flight autonomy (longer service life), low mobility (fewer changes in the topology), and better RSSI (better condition for data transmission).

FANETs are commonly employed to monitor regions by using sensors to capture images and/or videos. Therefore, it is very important that the quality of the video stream can be assessed using QoE metrics to ensure that, in fact, good data communication reflects a good user experience. Therefore, this paper performs a cross-layer evaluation involving the network and application layers to verify it.

\section{A Fuzzy Routing Protocol System}

The fuzzy system is a special kind of a knowledge-based system that works through dynamic and inaccurate sets of measurements. Fuzzy sets express the meaning of linguistic values related to a linguistic variable. A linguistic variable can be associated with a set of linguistic values, and that are associated with fuzzy sets that express the corresponding degree of relevance (truth value) of a linguistic variable. Fuzzy sets are used to quantify the corresponding degrees of uncertainty in evaluating a given concept (instance). Elements that belong to the fuzzy set may have degrees of relevance in the range of [0.1].

These systems are widely used in dynamic scenarios, such as vehicular network scenarios, being terrestrial or aerial because, as the dynamics occur in the scenarios, the values are changed, and, consequently, new solutions for the network are found. This paper considers three input metrics (language variables in the fuzzy system): mobility level, flight autonomy, and RSSI.

The level of mobility is linked to the speed metric, which is an important measure as it can affect the quality of communication/transmission because this measure indicates how fast the drones are moving, consequently changing the network topology as approach or distance from a particular area. For the variable, three linguistic values were defined: low speed (range 0 to $5 \mathrm{~m} / \mathrm{s}$ ), average speed (range 4 to $13 \mathrm{~m} / \mathrm{s}$ ), and high speed (when velocity is greater than $11 \mathrm{~m} / \mathrm{s}$ ).

Another important metric is the flight autonomy, directly linked to the battery capacity of the device; In this case, which is related to the time that the UAV will be able to fly over and monitor a particular region. The greater the battery capacity, the longer the flight range, and therefore, the longer the route, and network topology will remain active. This metric is divided into three linguistic values within this set: low range (0 to 600 seconds), medium 
range (600 to 1200 seconds), and high range (over 1200 seconds).

The third and last metrics used are linked to the signal strength, which can also represent the signal quality and is represented by the RSSI. The better the RSSI, the better the communication between two or more drones. Inversely, the worse the RSSI is, the worse is the signal quality between airborne devices, and the worse is the communication between them. In this metric, three language variables were defined for RSSI (dBi): low RSSI $(-125.1$ to $-102.1 \mathrm{dBi})$, average RSSI ( -111.1 to $63.1 \mathrm{dBi}$ ), and high RSSI (greater than -71.1).

With the support of the fuzzy system and the rule set used, accurate results will handle inaccurate and vague entries of input language variables, interpreted and classified as follows: terrible path, regular path, good path, and excellent path. When the drone detects a new path/route, it will provide the input variables collected for the embedded fuzzy system, which after applying the inference equation and the diffusion process, will indicate the quality level of the detected paths/routes, qualifying the best for the routing process of the air network.

In this paper, a Gaussian fuzzifier was used due to its inherent ability to reduce the noise of input variables [18]. Fuzzification is the mapping of input variables to a set of ranges that will be analyzed by a fuzzy rule base, the inference machine, and their respective membership functions of a set of linguistic variables, identifying which set it belongs to. After 100 interactions of the simulation process, it was concluded that the value of 0.6 would be considered for good or excellent paths. In certain situations, an inference value can be a part of two or more fuzzy sets at the same time (such as 0.55 ). In this case, the metric that has the most relevance according to the relevance function will be decisive in identifying the output set (Figure 2).

For the implementation and construction of this fuzzy system and after a set of tests and simulations, it was concluded that the ideal communication between drone devices would be in a scenario with high flight autonomy, high signal strength (RSSI), and low mobility level because in this scenario/topology, the route is kept longer so that the transmission stays longer and with fewer communication problems. The greater the distance from this ideal scenario, the fuzzy system can fetch the results that are closest to the context, based on a table of rules and the inference machine that can be dynamic and realistic in intelligent decisionmaking.

Table 2 shows a set of fuzzy system rules defined for performing this role. Through this set of rules and in conjunction with the implemented inference engine, the system finds the most appropriate decision to select the route/path that will remain active for the longest, improving and maintaining network performance with the best path (Table 2), mostly choosing the EXCELLENT and GOOD paths and very rarely the REGULAR and BAD paths.

The final decision is made according to the fuzzy system set inference values which qualify the highest inference value for the choice. During the implementation of the fuzzy system, it was observed that inference values equal to or

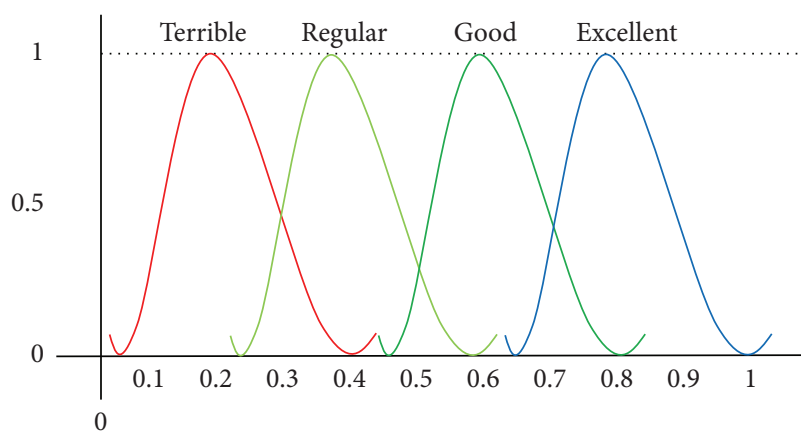

FIgURE 2: Output of the fuzzy system.

TABle 2: A fuzzy rule-based system.

\begin{tabular}{lccc}
\hline Mobility & Flight autonomy & RSSI & Output \\
\hline Low & Low & Low & Terrible \\
Low & Low & Medium & Regular \\
Low & Low & High & Regular \\
Low & Medium & Low & Regular \\
Low & Medium & Medium & Good \\
Low & Medium & High & Excellent \\
Low & High & Low & Regular \\
Low & High & Medium & Good \\
Low & High & High & Excellent \\
Medium & Low & Low & Terrible \\
Medium & Low & Medium & Regular \\
Medium & Low & High & Regular \\
Medium & Medium & Low & Regular \\
Medium & Medium & Medium & Good \\
Medium & Medium & High & Excellent \\
Medium & High & Low & Regular \\
Medium & High & Medium & Good \\
Medium & High & High & Excellent \\
High & Low & Low & Terrible \\
High & Low & Medium & Terrible \\
High & Low & High & Terrible \\
High & Medium & Low & Terrible \\
High & Medium & Medium & Regular \\
High & Medium & High & Regular \\
High & High & Low & Terrible \\
High & High & Medium & Regular \\
High & High & High & Regular \\
\hline & & &
\end{tabular}

higher than 0.6 represented the best way in these scenarios and therefore those with the highest probability of being chosen (Figure 3).

In the 3D graphic image of Figure 4, the yellow areas represent the routes/paths most likely to be chosen, as they correspond to routes where drones have high flight autonomy, low mobility, and high RSSI. The green-shaded chart area corresponds to the average speed, average RSSI, and average flight range drones, and in this situation, there is little chance of the drone being chosen as the communication route. The last region, represented by the blue color on the chart, represents a drone with high mobility, low RSSI, and low flight range, in which case the drone will not be chosen as the communication route (Figure 4). 

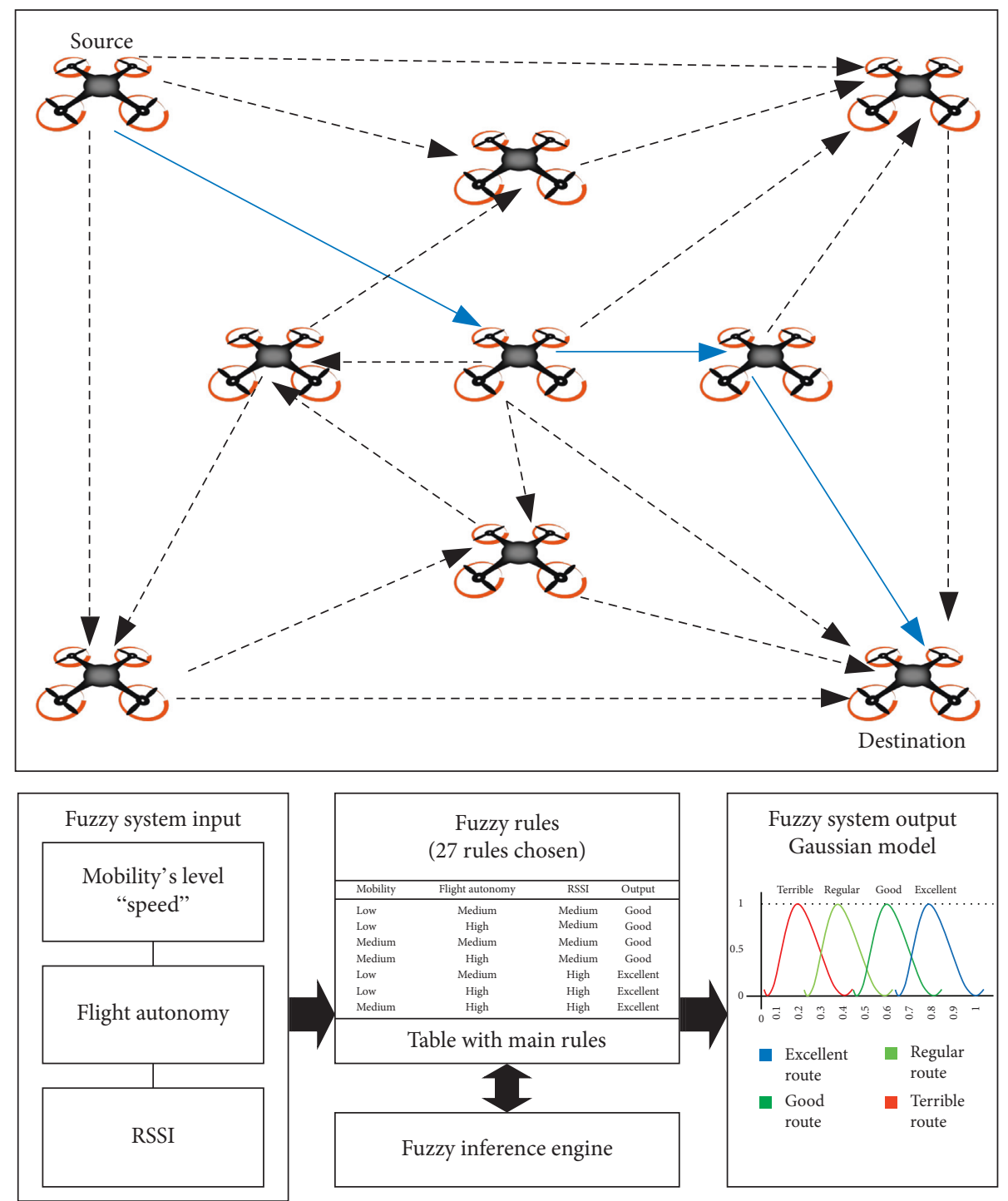

FIgURE 3: Schematic representation of the proposal.

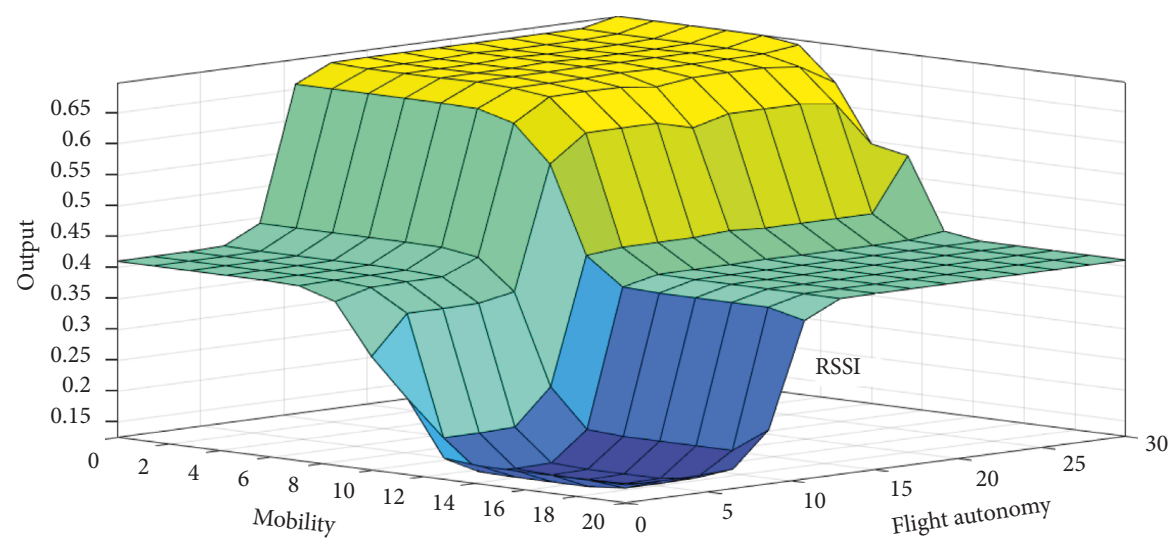

FIGURE 4: Fuzzy inference system.

\section{Results}

This section evaluates the performance of the FANET protocol by proving the benefit of the proposed routing protocol when compared to AODV (reactive protocol) and OLSR (proactive protocol). The results were performed by simulation through the network simulator version 2 and multimedia applications. The video used was the "Sintel" 
TABLE 3: NS-2 simulation parameters.

\begin{tabular}{lr}
\hline Drones & 10 \\
\hline Access technology & IEEE $802.11 \mathrm{~g}$ \\
Propagation model & Shadowing \\
Mobility type & Random waypoint \\
Query & Droptail \\
Number of simulations & 100 \\
Confidence interval & $95 \%$ \\
Frequency & $2.4 \mathrm{GHz}$ \\
Area & $200 \mathrm{~m} \times 200 \mathrm{~m}$ \\
\hline
\end{tabular}

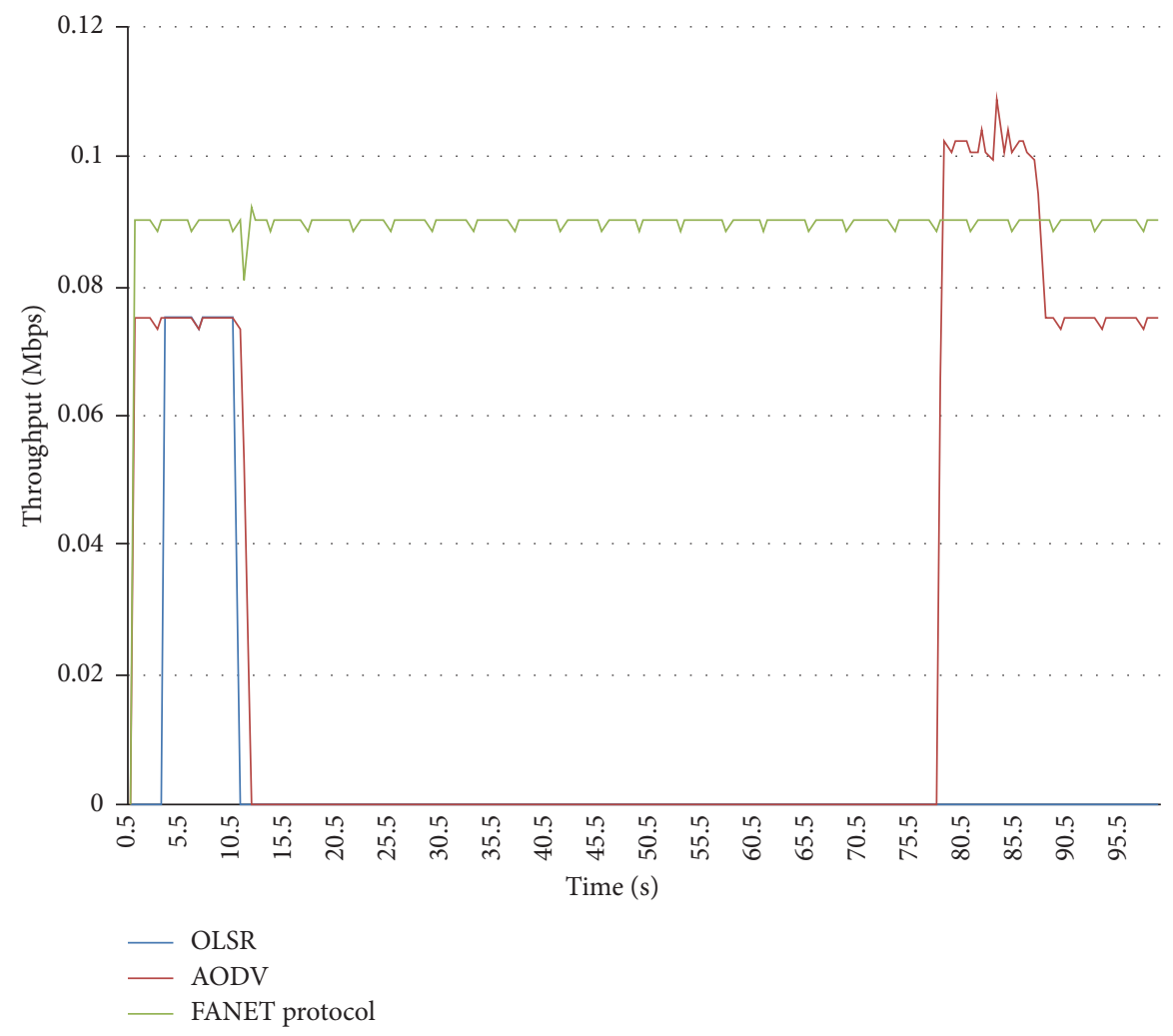

Figure 5: Throughput over time.

which is high definition (HD $1280 \times 7200$ p) with 1200 frames.

The propagation model used in the simulations was shadowing because it is more realistic in signal attenuation than the free-space model and two-ray ground models (propagation models available in NS-2) [19]. The simulator does not support three-dimensional scenarios, so it is assumed that drones are at similar heights and with a line of sight of communication. Table 3 shows the simulation parameters used for scenarios with 10 drones with random mobility (random way point) and speed range from $2 \mathrm{~m} / \mathrm{s}$ to $20 \mathrm{~m} / \mathrm{s}$ in an area of $200 \mathrm{~m} \times 200 \mathrm{~m}$.

Due to the drones' random mobility, they can fly closer or farther from each other, considering the speed drones can also get closer or fly slower or faster from each other. Changes in the network topology due to drone mobility require a fast response from routing protocols; if this response does not occur efficiently, network performance will be degraded.
The FANET adaptive protocol outperformed other protocols as shown in Figure 5. The AODV and OLSR protocols, due to network topology changes, did not update their routing tables quickly and efficiently and were unable to maintain an active route, and consequently, both protocols interrupted their transmissions during the part of the simulation. Unlike the others, the FANET adaptive protocol updated its routing table quickly and efficiently always keeping an active route for transmission and consequently avoiding connection breakdown.

The throughput average also shows that the FANET adaptive protocol performed better when compared to AODV and OLSR protocols. The proposed routing protocol performed approximately $300 \%$ better than the other two protocols, as shown in Figure 6.

In addition to evaluating through quality of service metrics, this paper also evaluated the FANET adaptive protocol using the quality of experience metric. The three main QoE metrics are peak signal-to-noise ratio (PSNR), 


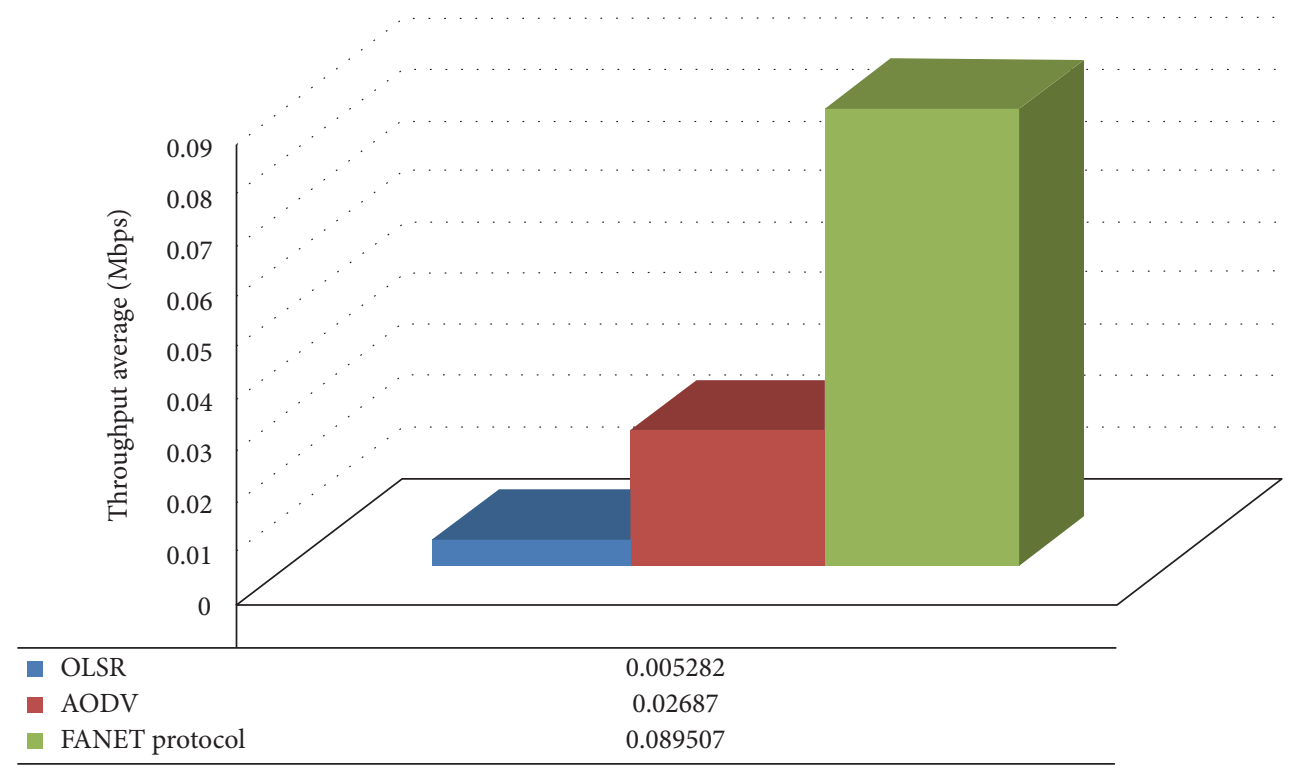

Figure 6: Average throughput.

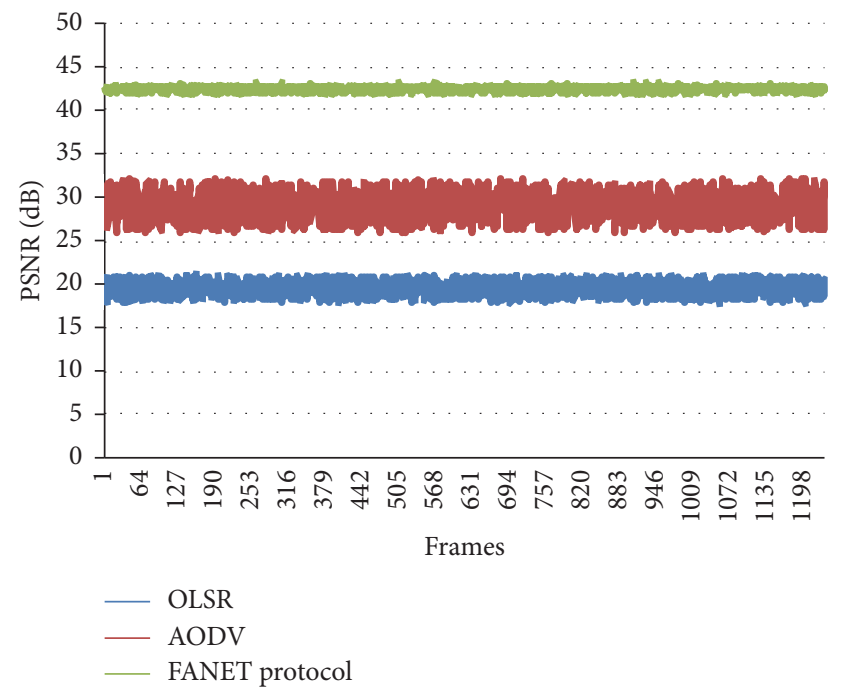

Figure 7: PSNR values.

structural similarity (SSIM), and video quality metric (VQM). PSNR evaluates the signal-to-noise ratio of the video considering features such as brightness, noise, and color. Figure 7 shows that the OLSR protocol had a PSNR average of $18 \mathrm{~dB}$ (rated as the poor video), the AODV protocol had a PSNR average of $28 \mathrm{~dB}$ (rated as the regular video), and the FANET adaptive protocol had an average PSNR of $42 \mathrm{~dB}$ (rated as the excellent video).

The FANET adaptive protocol also outperformed OLSR and AODV when evaluated using the SSIM metric. The metric evaluates the video considering features such as brightness, color, and contrast. The SSIM metric has a value scale from 0 to 1 , the closer to value 1 , the better the video quality. The OLSR protocol had an average SSIM of 0.43 (considered a bad video), the AODV protocol had an average SSIM of 0.61 (considered a regular video), and the

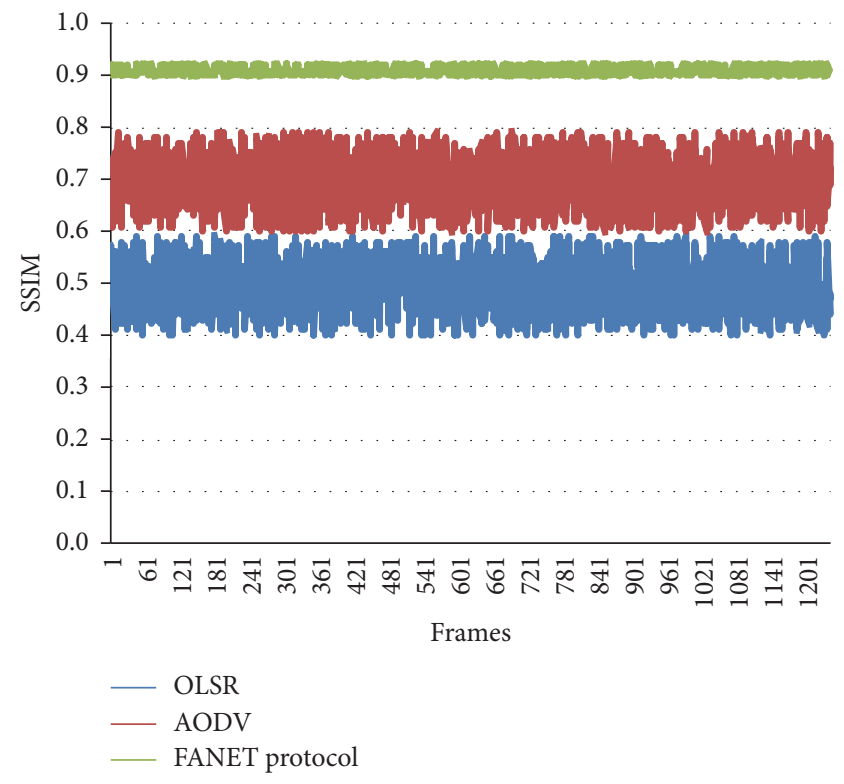

Figure 8: SSIM values.

FANET adaptive protocol had an average SSIM of 0.91 (considered an excellent video), as shown in Figure 8.

The comparison between the three protocols was also made using the VQM metric, which also showed the superiority of the FANET adaptive protocol. The VQM metric has a scale from 0 to 5 , in which case the closer to 0 , the better the video quality. The metric evaluates the video considering features such as color, brightness, intensity, and frame distortion. The OLSR protocol had a VQM average of 4.1, the AODV protocol had a VQM average of 3.3, and the FANET adaptive protocol had a VQM average of 1.4 as shown in Figure 9.

This paper also evaluated the protocols by visually comparing frames received using the MSU Video Quality 


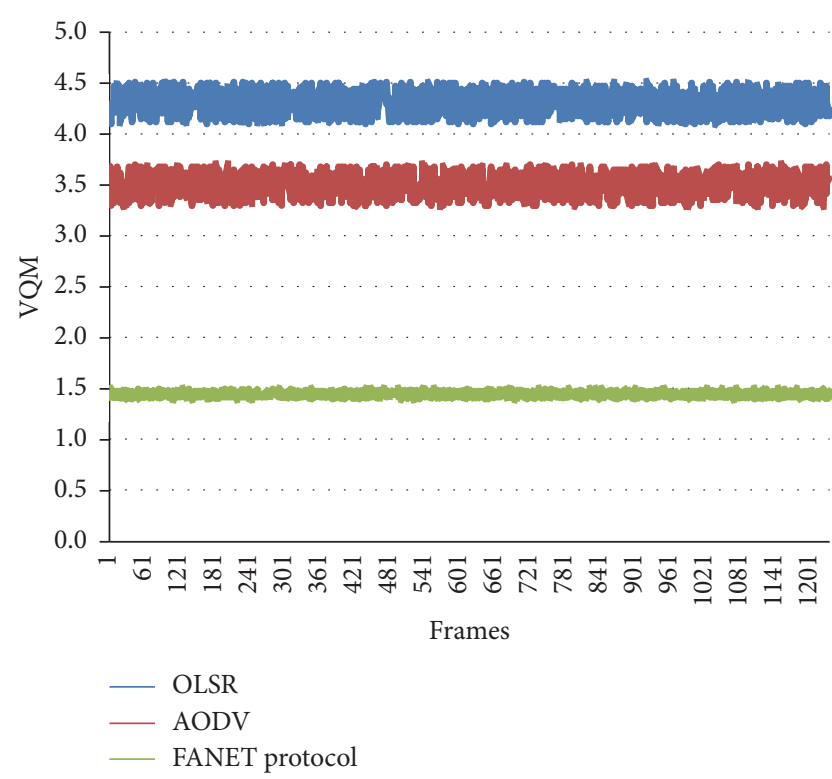

FIGURE 9: VQM values.

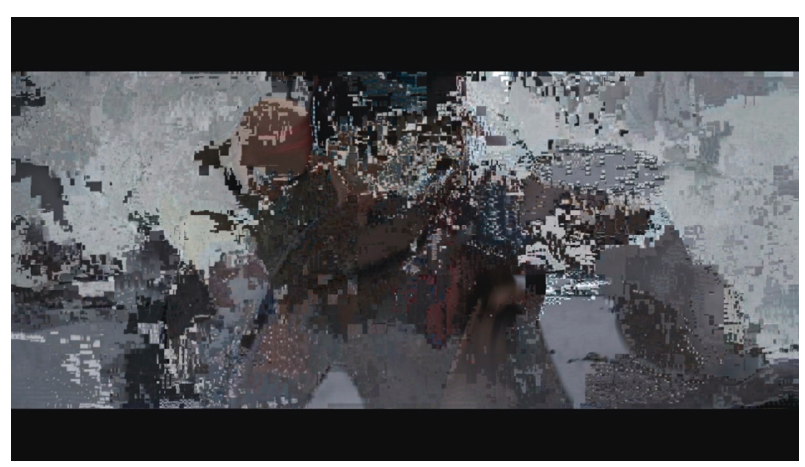

Figure 10: Frame received by OLSR.

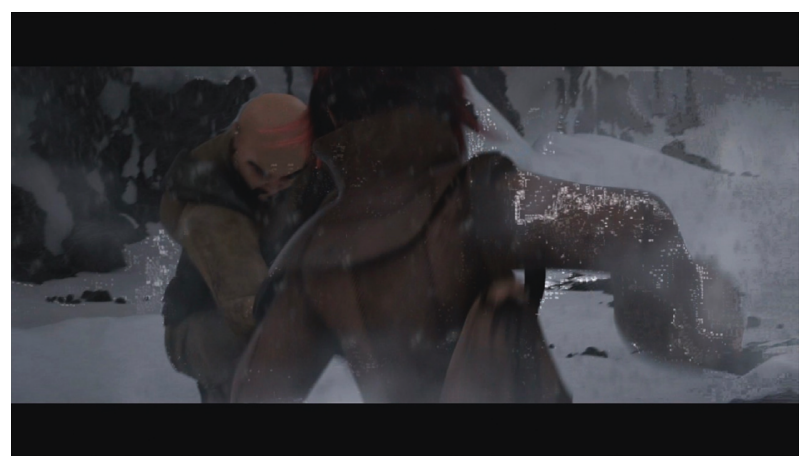

Figure 11: Frame received by AODV.

Measurement Tool software [20]. Visual comparison of frames transmitted by each protocol also showed better performance of the FANET adaptive protocol. Figure 10 shows a video frame transmitted by the OLSR protocol in which it is possible to observe that the frame is distorted and has pixel flaws.

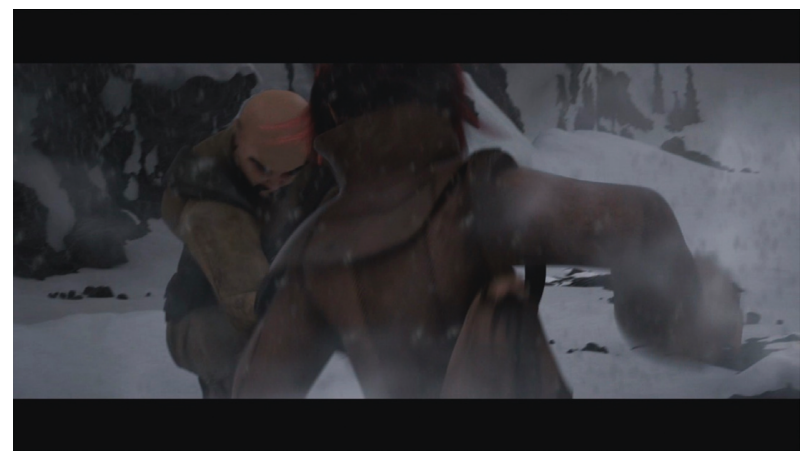

FIGURE 12: Frame received by the fuzzy system-based routing protocol.

Figure 11 shows a video frame transmitted by the AODV protocol, in which there was a quality improvement when compared to the previous frame. However, the video cannot be rated as of excellent quality, and it has minor distortions and flaws, so it is classified as a regular quality video.

The video frame transmitted by the FANET adaptive protocol has a better quality when compared to AODV and OLSR protocols. Figure 12 shows that the frame has no distortions or flaws being rated as an excellent quality video.

\section{Conclusion}

FANETs provide several benefits (as cited earlier), but there are still some challenges to be studied, such as routing between UAVs in the air network. For this reason, the flying networks have been a research topic.

There is no specific routing protocol for air networks, so this paper proposes a routing protocol for FANETs that uses a fuzzy system to improve the route discovery process. The FANET adaptive protocol takes into account RSSI, mobility level, and flight autonomy.

The FANET adaptive protocol was compared with AODV and OLSR protocols (traditional ad hoc routing protocols). The comparison was carried out by simulation using QoS and QoE metrics, and the proposed routing protocol had a better performance (around 35\%) than the other two routing protocols.

In future paper, the authors intend to develop new artificial intelligence techniques and include new parameters for decision machine, as well as use a new wireless technologies (i.e., long-term evolution-LTE) and new propagation models (i.e., air-to-ground path loss for low-altitude platforms and air-to-ground path loss for high-altitude platforms).

\section{Data Availability}

The data used to support the findings of this study are available from the corresponding author upon request.

\section{Conflicts of Interest}

The authors declare that they have no conflicts of interest. 


\section{References}

[1] E. Cruz, "A comprehensive survey in towards to future FANETs," IEEE Latin America Transactions, vol. 16, no. 3, 2018.

[2] O. Burdakov, P. Doherty, K. Holmberg, J. Kvarnström, and P.-M. Olsson, "Relay positioning for unmanned aerial vehicle surveillance," The International Journal of Robotics Research, vol. 29, no. 8, pp. 1069-1087, 2018.

[3] Phantom 4 user manual V 1.6, 2017, https://dl.djicdn.com/ downloads/phantom_4/20170706/Phantom_4_User_ Manual_v1.6.pdf.

[4] Mavic 2 pro zoom user manual V 2.0, 2019, https://dl.djicdn.com/ downloads/Mavic_2/20190417/Mavic_2_Pro_Zoom_User_ Manual_v2.0_en.pdf.

[5] S. Winkler, "Perceptual video quality metrics-a review," in Digital Video Image Quality and Perceptual Coding, CRC Press, Boca Raton, FL, USA, 2005.

[6] Z. Zheng, A. Sangaiah, and T. Wang, "Adaptive communication protocols in flying ad hoc network," IEEE Communications Magazine, vol. 56, no. 1, pp. 136-142, 2018.

[7] D. S. Vasiliev, D. S. Meitis, and A. Abilov, "Simulation-based comparison of AODV, OLSR and HWMP protocols for flying ad hoc networks," in Proceedings of the Internet of Things, Smart Spaces, and Next Generation Networks and Systems NEW2AN 2014, S. Balandin, S. Andreev, and Y. Koucheryavy, Eds., pp. 245-252pp. 245-, St. Petersburg, Russia, August 2014.

[8] F. Khelifi, A. Bradai, K. Singh, and M. Atri, "Localization and energy-efficient data routing for unmanned aerial vehicles: fuzzy-logic-based approach," IEEE Communications Magazine, vol. 56, no. 4, pp. 129-133, 2018.

[9] A. Nayyar, "Flying adhoc network (FANETs): simulation based performance comparison of routing protocols: AODV, DSDV, DSR, OLSR, AOMDV and HWMP," in Proceedings of the International Conference on Advances in Big Data, Computing and Data Communication Systems (icABCD), pp. 1-9, Durban, South Africa, August 2018.

[10] P. Kaur and A. Singh, "Nature-inspired optimization techniques in VANETs and FANETs: a survey," in Advanced Computational and Communication Paradigms. Advances in Intelligent Systems and Computing, S. Bhattacharyya, N. Chaki, D. Konar, U. Chakraborty, and C. Singh, Eds., vol. 706, Springer, Singapore, 2018.

[11] G. A. Litvinov, A. V. Leonov, and D. A. Korneev, "Applying static mobility model in relaying network organization in mini-UAVs based FANET," in Proceedings of the 2018 Systems of Signal Synchronization, Generating and Processing in Telecommunications (SYNCHROINFO), pp. 1-7, Minsk, Belarus, July 2018.

[12] O. S. Oubbati, A. Lakas, F. Zhou, M. Güneș, and M. B. Yagoubia, "A survey on position-based routing protocols for Flying Ad hoc Networks (FANETs)," Vehicular Communications, vol. 10, pp. 29-56, 2017.

[13] D. Radu, A. Cretu, B. Parrein et al., "Flying ad hoc network for emergency applications connected to a fog system," in Advances in Internet, Data \& Web Technologies, pp. 675-686, Fev, Aachen, Germany, 2018.

[14] H. Yang and Z. Liu, "An optimization routing protocol for FANETs," J Wireless Com Network, vol. 2019, no. 1, p. 120, 2019.

[15] W. Zafar and B. Khan, "Flying ad-hoc networks: technological and social implications," IEEE Technology and Society Magazine, vol. 35, no. 2, pp. 67-74, 2016.
[16] S. Rosati, K. Krużelecki, G. Heitz, D. Floreano, and B. Rimoldi, "Dynamic routing for flying ad hoc networks," IEEE Transactions on Vehicular Technology, vol. 65, no. 3, 2016.

[17] B. Yang, M. Liu, and Z. LI, "Rendezvous on the fly: efficient neighbor discovery for autonomous UAVs," IEEE Journal on Selected Areas in Communications, vol. 36, no. 9, 2018.

[18] Z. Ju and H. Liu, "Fuzzy Gaussian mixture models," Pattern Recognition, vol. 45, no. 3, 2012.

[19] The ns manual (formerly ns notes and documentation), 2011, https://www.isi.edu/nsnam/ns/doc/ns_doc.pdf.

[20] MSU quality measurement tool, 2016, https://www.compression. ru/video/quality_measure/vqmt_download.html. 


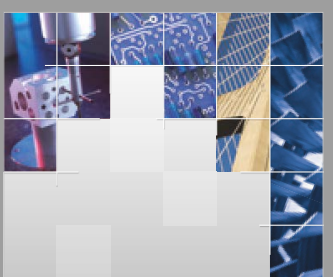

\section{Enfincering}
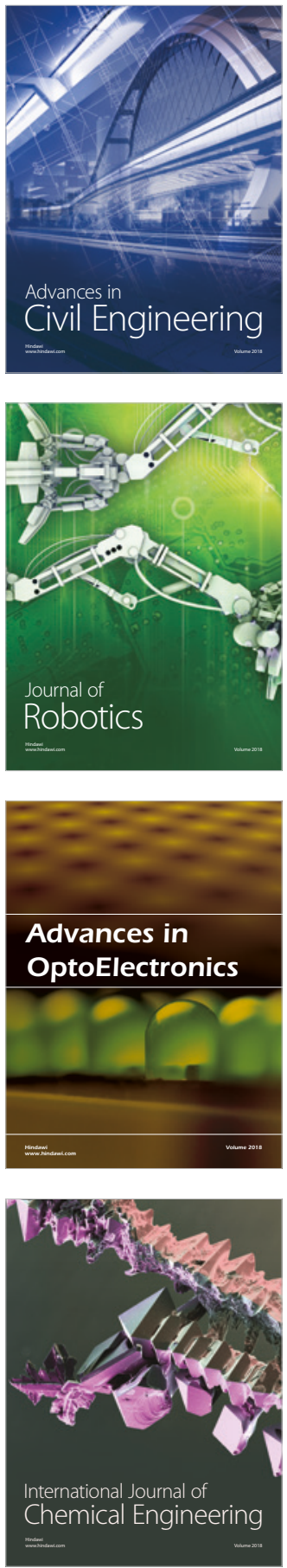

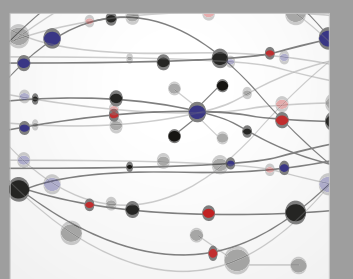

\section{Rotating \\ Machinery}

The Scientific World Journal

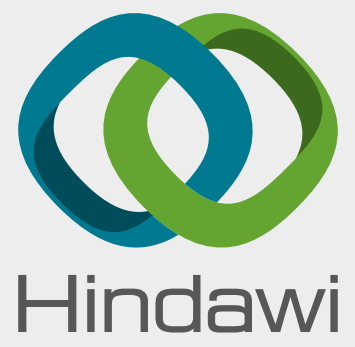

Submit your manuscripts at

www.hindawi.com
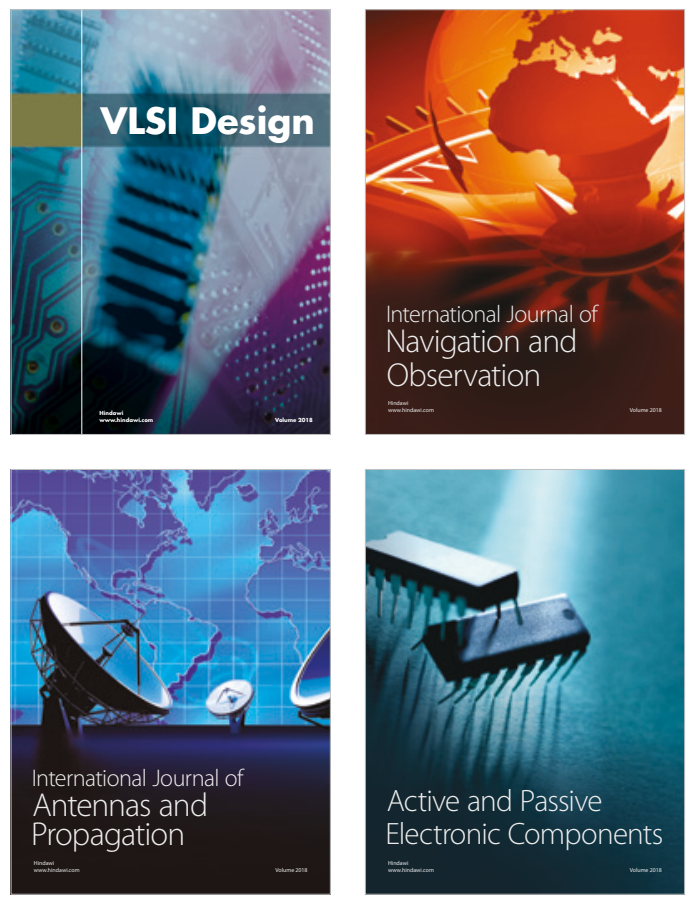
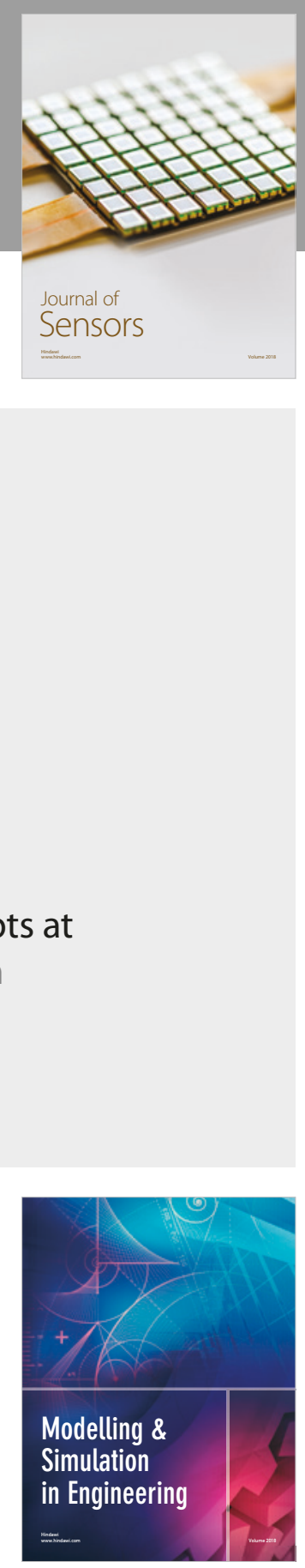

\section{Advances \\ Multimedia}
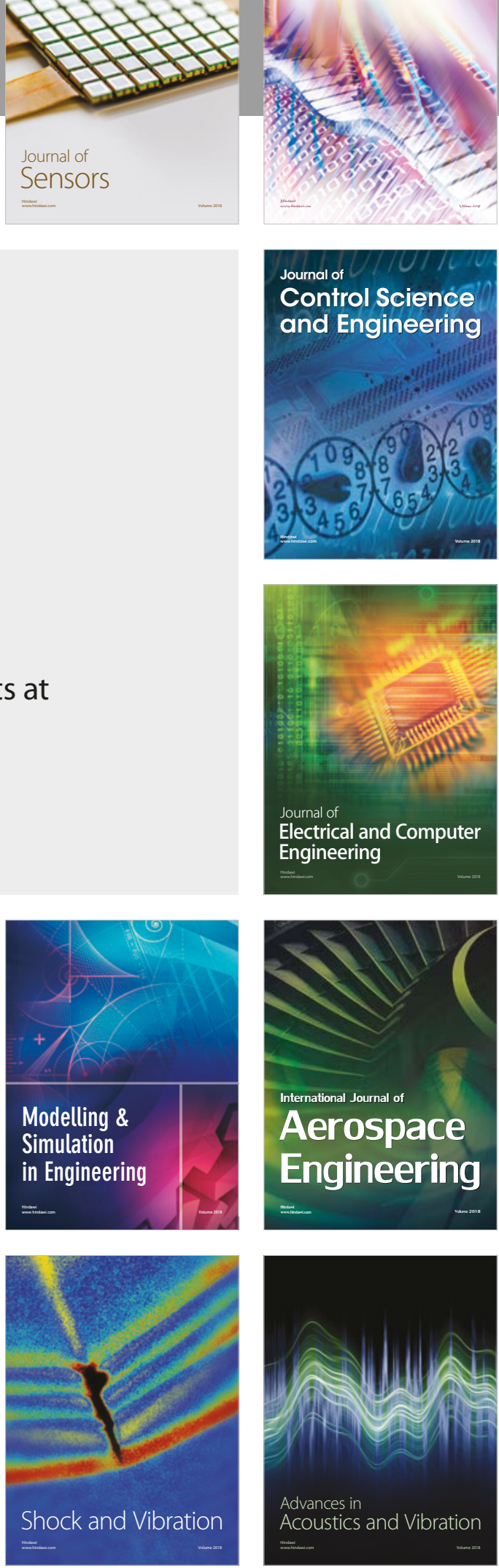